\title{
SYNCHRONOUS RELAXATION FOR PARALLEL ISING SPIN SIMULATIONS
}

\author{
Boris Lubachevsky \\ bdl@bell-labs.com \\ Alan Weiss \\ apdoo@bell-labs.com \\ Hill, New Jersey, USA
}

\begin{abstract}
A new parallel algorithm for simulating Ising spin systems is presented. The sequential prototype is the $n$-fold way algorithm [2], which is efficient but is hard to parallelize using conservative methods. Our parallel algorithm is optimistic. Unlike other optimistic algorithms, e.g., Time Warp, our algorithm is synchronous. It also belongs to the class of simulations known as "relaxation" [3] hence it is named "synchronous relaxation." We derive performance guarantees for this algorithm. If $N$ is the number of PEs, then under weak assumptions we show that the number of correct events processed per unit of time is, on average, at least of order $N / \log N$. All communication delays, processing time, and busy waits are taken into account.

Key words: conservative simulation, optimistic simulation, computational physics, Metropolis algorithm
\end{abstract}

\section{Introduction}

The Ising model of computational physics was introduced in 1925 [7] for describing magnetization phenomena. It has since been in use both for its original purpose, and as a computational metaphor in areas ranging from economics [10 to wireless communications [1 4] to material science [16.

In a simple version of the model we have a planar lattice of atoms, each of which may be in one of two spin states, +1 or -1 . The atoms flip their spins stochastically in a way that depends only on the states of their nearest neighbors, on an external magnetic field, and on temperature.

Ising spin simulations have always been slow. There have been a number of proposals for speeding them up. The fastest available serial algorithm that does not violate the stochastic properties of the procedure by Metropolis et al. [14] is the $n$-fold way algorithm [2]. A parallel version of the $n$-fold way was proposed in [11, 12] and implemented in [9]. Its speedup with respect to the serial $n$-fold way algorithm was not very high in low temperature. This was because at low temperature the algorithm performed many tentative calculations of flips that were rejected at the end. In the procedure atoms along the boundaries of regions hosted by different processing elements were subject to such rejections; only atoms in the interior of regions were not. As the rejection rate asymptotically approaches $100 \%$, even if the fraction of atoms on the boundaries is small, the rejection overhead becomes the dominant bottleneck in simulation. Note that the main advantage of the $n$-fold way over the procedure in [14] is absence of rejections.

The parallel algorithms in 11, 12 are conservative. Herein we describe a new algorithm for Ising spin simulations whose aim is to eliminate the drawbacks associated with rejections. The algorithm is optimistic; it belongs to the class of "relaxation" simulations [3]. It is also a synchronous algorithm, and as such, it differs from Time Warp [8]. Our "synchronous relaxation" implements the $n$-fold way algorithm in parallel by repeatedly generating a time segment of the spin flip trajectory. After each iteration, the PEs exchange information they generated, and correct the errors thus revealed. Unlike conservative schemes in 11, 12, computations are not wasted on rejections. And unlike Time Warp, erroneous events are corrected in a synchronous, iterative fashion rather than asynchronously. However, iterations might introduce extra computations.

Since Time Warp is asynchronous, and our algorithm is not, the behavior and analyses of these algorithms are quite different. In our analysis we estimate 
the number of iterations needed to correct mistakenly simulated events and the amount of work in each iteration. Under weak assumptions, we show that the former is small and the latter is not excessive. Synchronous relaxation was discussed in 4 in a different context, and without formal proofs.

Note that Metropolis algorithm in 14 simulates a certain Markov Chain. Markov Chain uniformization is put forward in 6, 15] as a tool for parallelizing a sequential Markov Chain simulation. Algorithms in 11. 12 turn out to be a specialization of more general schemes in [6]. It should be noted that the actual development of Ising spin simulations did not follow this logic. Specifically, the rejection scheme 14, was proposed as an inherently serial algorithm, without any reference to parallel processing or uniformization. Then scheme 2] was proposed as a derivative of, and an improvement over 14, since it got rid of event rejections. Note that according to 6, 15 it is the scheme in 14 that would rather be considered a derivative of [2], the derivative which is more amenable to parallelization. Actually, it took a while [11, 12] to break the tradition of thinking the scheme 14] as being inherently serial. Optimistic methods of parallelization of Markov Chain simulations are discussed in 15, but still in connection with uniformization, i.e., with event rejections. Since our present synchronous relaxation method for parallelization of the scheme in 2] eliminates rejections, it apparently does not follow from [15]; its efficiency analysis is certainly new.

\section{The Ising model}

In the model atoms are located at the vertices of a rectangular subset $G$ of an orthogonal lattice. A configuration is defined by the spin variables $s(v)= \pm 1$ for atoms $v \in G$. In accordance with [14, the time evolution of the configuration is a sequence of single spin updates: given a configuration, define the next configuration by choosing a vertex $v$ uniformly at random and changing $s(v)$ to $-s(v)$ with independent probability $p$, computed as described in the next paragraph. With probability $1-p$, the $s(v)$ remains unchanged.

Computing the probability $p=p(v)$ involves the 4 nearest neighbors of vertex $v=(i, j):(i+1, j),(i-$ $1, j),(i, j+1),(i, j-1)$. Periodic boundary conditions are assumed in both directions. Computing the $p(v)$ also involves the Hamiltonian

$$
E=-J \sum_{v, v^{\prime} \in G} s(v) s\left(v^{\prime}\right)-H \sum_{v} s(v)
$$

where $J>0$ and $H$ are constants, and the pairs $\left(v, v^{\prime}\right)$ of indices in the first sum are nearest neighbors. The value of $p$ is given in [14] by

$$
p=\min \{1, \theta\}, \quad \theta=\exp \left(-\Delta E / k_{B} T_{\text {abs }}\right)
$$

where $\Delta E=\Delta E(v)$ is the energy change that would result from the flip of the spin at site $v, k_{B}$ is Boltzmann's constant, and $T_{\text {abs }}$ is the absolute temperature. In [5] a different expression for $p$ is used:

$$
p=\theta /(1+\theta)
$$

with the same $\theta$. Note that computing $\Delta E(v)$ for spin $v$ involves only the values of $s(v)$ and $s\left(v^{\prime}\right)$ for the nearest neighbors $v^{\prime}$ of $v$. According to (2.2), a spin flip which results in lower energy, $\Delta E<0$, certainly occurs, because in such a case, $p=1$; but in (2.3) $p<1$ always and even the flips with $\Delta E<0$ can sometimes be rejected.

\section{The $n$-fold way algorithm}

We suppose that attempts of a flip by each atom constitute an independent Poisson process ${ }^{1}$ with fixed rate, say $\lambda$. Then the successful attempts is also a Poisson process, but with a smaller rate $\lambda p, p=p(t)$, which, in general, varies in time.

The $n$-fold way algorithm in 2 splits all atoms into $n$ classes, where a class is defined as those atoms with the same flip probability $p$, given either by (2.2) or (2.3). In the 2D Ising model there will be no more than $n=10$ classes: each atom may be either up or down, and its four neighbors have 5 possibilities (none up, one up,..., 4 up), so there are $2 \times 5$ possible configurations for each atom For each class $k$ define

$$
\begin{aligned}
N_{k} & =\text { number of atoms in class } k \\
p_{k} & =\mathbb{P} \text { (atom in class } k \text { flips when chosen }) .
\end{aligned}
$$

Given that the Poisson rate of flips of a single atom of class $k$ is $\lambda p_{k}$, the combined rate of flips of all atoms is

$$
\Lambda=\lambda \sum_{k} N_{k} p_{k}
$$

\footnotetext{
${ }^{1}$ In $2 \longdiv { 1 4 }$ system state changes are just a sequence. For the sake of parallelization we need spin flips to be a Poisson process in continuous time. This assumption is consistent with the models in 2 14, but is not mentioned there.
} 
and the combined process whose arrivals coincide with spin flips at any atom also enjoys the Poisson property. As spins flip, membership of atoms may change, $N_{k}$ may vary in time $N_{k}=N_{k}(t)$, and so may the Poisson rate of the combined process $\Lambda=\Lambda(t)$.

It follows that given the time $\tau_{i-1}$ of the $(i-1)$ th flip, the time of the $i$ th flip can be generated as

$$
\tau_{i}=\tau_{i-1}+\frac{-\log U_{i}}{\Lambda\left(\tau_{i-1}\right)},
$$

where $U_{i}$ is the $i$ th independent sample of a random $(0,1)$ uniformly distributed variable. The first spin flip time $\tau_{1}$ can be generated as in 3.4 with $i=1$ if we formally set $\tau_{0}=0$.

We should then select the atom whose spin is to flip. This is done in three phases. In Phase 1 we generate an independent random $(0,1)$ uniformly distributed variable $V_{i}$. In Phase 2 we choose a class $k_{*}$ to which the atom to be flipped belongs. This is done by linearly scanning the sequence of classes $k=1,2, \ldots n$ while summing the weights of their chances to be selected

$$
W(k)=\sum_{j=1}^{k} \frac{N_{j} p_{j}}{\sum_{i} N_{i} p_{i}} .
$$

Once the inequality

$$
W(k-1)<V_{i} \leq W(k)
$$

is detected for $k=k_{*}$, we stop.

In Phase 3 we choose an atom in class $k_{*}$. All atoms in each class are maintained in a linear order. To determine the index of an atom in the order we take the normalized residual $R_{i}$ of the random sample $V_{i}$. This is defined as

$$
R_{i}=\frac{V_{i}-W\left(k_{*}-1\right)}{\frac{N_{k_{*}} p_{k_{*}}}{\sum_{j} N_{j} p_{j}}},
$$

where $k_{*}$ is the class index found in Phase 2. The required index is then

$$
\text { access index }=\left\lfloor R_{i} N_{k_{*}}\right\rfloor+1 .
$$

Note that each spin flip changes the class membership of several atoms. We omit here the discussion of an elaborate data structure 2] which maintains the classes in the computer memory so that the atoms in the classes are positioned in the required linear order. We note that it only takes $O(1)$ computations to adjust this order at each spin flip. Experiments 2] confirm that at a low temperature, the $n$-fold way algorithm is much faster than the original Metropolis et al. algorithm with rejections in [14].

\section{Synchronous relaxation: a general formulation}

Suppose that a simulation of a system is to be performed on a multiprocessor with $N$ processing elements (PEs). Our procedure is to give each PE a subsystem to host, and have the PE produce the history of that subsystem.

Each PE will keep track of the simulated time before which all simulated histories are known; this quantity is called committed time. Committed time increases in steps, in synchrony among all PEs; its value is common to all PEs. Each step consists of several iterations. At each iteration, each PE produces a tentative or speculative history of the subsystem it hosts beyond the current committed time. The PE extends its local history until its local time reaches the committed time plus $T_{\max }$, where $T_{\max }$ is the step size of committed time increases. Since subsystems are, in general, connected, in order to produce a correct local history, a $\mathrm{PE}$ needs to know the correct local histories of other PEs. But they are not known, because other PEs are in the same quandary. If, by some miracle, a PE knew the correct histories of all others, then it would produce a correct history for itself; when all PEs produce correct histories, then the next committed time will become committed time plus $T_{\max }$. So all we have to explain is how to achieve this miracle.

The mechanism is by iterations. During the first iteration, each PE makes the simplest assumption about the histories of the other PEs; we call this the canonical assumption. For example, it may assume that the states of the other subsystems do not change. This assumption will enable it to produce its own history. After all the PEs generate local history for an additional $T_{\max }$ units of simulated time, they compare their histories. This comparison is done in synchrony, in the sense that the PEs perform the comparison only after they have all completed the previous task of generating their histories. As a rule, there will be inconsistencies between the assumed and actual (generated) histories of other PEs. If so, the PEs need to perform more iterations.

During subsequent iterations, if a PE needs to know the local history of another PE, it uses that history generated in the last iteration. The goal of producing correct histories at a step is achieved if no $\mathrm{PE}$ detects any inconsistencies between the assumed and actual history of any other PE. Once this hap- 
pens, all PEs increase committed time by $T_{\max }$, and continue.

We now give a representation of the synchronous relaxation algorithm. In the outset of the simulation, committed time is set to zero. The subsystems hosted by the PEs are set to their initial states. Then each PE executes the following code. The execution is asynchronous except for the two "synchronize" statements. While executing a "synchronize," each PE waits for the other PEs to reach the same statement.

DO (step)

1. Choose a step size $T_{\max }$ for committed time increase.

2. Make canonical assumption about other PEs' histories.

DO (iteration)

(a) Generate local history, starting with committed time until local time $\geq$ committed time $+T_{\max }$.

(b) Synchronize.

(c) Compare generated history of other PEs with the corresponding assumptions. If they differ, replace the current assumptions with the corresponding histories.

(d) Synchronize.

UNTIL (iteration) all PEs detect no difference in the comparison in step $2 \mathrm{C}$

3. Increment committed time by $T_{\max }$.

UNTIL (step) committed time exceeds a prespecified value.

There are two issues that need to be addressed. Will there ever come a time when no inconsistencies are detected? Another is the question of correctness. Is the lack of inconsistencies equivalent to correctness of the history? Generally, the iterations will not terminate. However, when applied to discrete event simulations, under mild conditions we can show that the iterations do terminate. Also, under weak conditions, we can show that lack of inconsistency is equivalent to correctness (see 13]).

The preceding algorithm turns out to be applicable not only to deterministic simulations, but to stochastic simulations, too, using the following technique. Sequences of (pseudo)random numbers are employed in stochastic simulations. We should treat these sequences as deterministic. To this end, all we have to do is to reuse the (pseudo)random numbers generated at an iteration, during the course of the following iterations. An extreme way of effecting this is to generate all random numbers in a list before the rest of the simulation begins. This is a helpful way to think about simulation, whether or not it turns out to be a practical method in any instance.

The general description above may not give enough details for a specific implementation. Furthermore, it may not be clear how to estimate the efficiency of the algorithm; specifically, how to bound the number of iterations in each step. The next sections will fill in these details for the Ising model.

\section{Synchronous relaxation for Ising simulation}

In this section we detail the synchronous relaxation algorithm for Ising simulation using the $n$-fold way algorithm. While simulations can be implemented correctly even if different PEs run different serial algorithms to generate local history, we are interested in high performance, so we will concentrate on a parallelization of the most efficient serial algorithm.

We follow the algorithm outlined in Section 4 The set $G$ is partitioned into subsets $G_{i}, 1 \leq i \leq N$, and each $G_{i}$ is hosted by a separate processing element, $\mathrm{PE}_{i}$. Each $G_{i}$ corresponds to a subsystem in the general description of Section 4 As before, the boundary of $G_{i}$ is denoted $\partial G_{i}$.

Statement 1 of the general relaxation procedure requires a choice of step size $T_{\max }$ for each committed time increase. In the simplest algorithm formulation, we choose the same step value to serve for all committed time increase steps,

$$
T_{\max }=\frac{\log N}{\lambda\left|\partial G_{i}\right|},
$$

assuming that all the sizes of boundary regions $\left|\partial G_{i}\right|$ are equal, and where $\lambda$, as in Section 3 is the largest rate at which any atom can flip. 
Statement 2 of the general relaxation procedure requires each $\mathrm{PE}$ to make a canonical assumption concerning its neighbors' histories. We choose the assumption that no spin flip occurs in any neighboring atom during the initial committed time increase step $T_{\max }$.

Statement (a) requires each $\mathrm{PE}$ at each iteration to generate a segment of local history. The segment begins at the current committed time value, let us call it $t_{c}$, and ends at time $t_{c}+T_{\max }$. The history can be identified with a sequence $t_{c} \leq \tau_{1}<\tau_{2}<\ldots<\tau_{m}<$ $t_{c}+T_{\max }$ of spin flip times and the corresponding sequence of atoms $v_{1}, v_{2}, \ldots v_{m}$ that flip their spins at these times. Because the procedure is complex and subtle, our discussion will be lengthy. We split it into several cases.

First iteration, first step. Thanks to the canonical assumption, the procedure executed by each $\mathrm{PE}$ in this case is almost a literal repetition of the sequential algorithm described in Section [3] As in that algorithm, a sequence of pairs $\left(U_{1}, V_{1}\right),\left(U_{2}, V_{2}\right), \ldots,\left(U_{m}, V_{m}\right)$, of independent samples of uniform $(0,1)$ distributed random variables $U_{i}$ and $V_{i}$, feeds the generation of the spin flip history. The $U_{i}$ feeds formula (3.4), which generates $\tau_{i}$, given $\tau_{i-1}$ and given the previous system state. The $V_{i}$ feeds the procedure that selects the class of the atom $v_{i}$ to flip and selects the $v_{i}$ 's index according to formulas (3.7) and (3.8). To compute $\tau_{1}$ using (3.4) for $i=1$ we formally assume $\tau_{0}=0$. As in the sequential case, this can be done, because the state of the system at time $t_{c}=0$ is known. The computations continue for as long as $\tau_{i}$ computed by (3.4) is smaller than $t_{c}+T_{\max }$. The last $\tau_{i}$ that satisfies this condition becomes $\tau_{m}$.

Steps (b), (c), and (d), that follow are obvious. Note that while comparing the generated histories with the assumed histories, as required in step (c), the $\mathrm{PE}$ pays attention only at the single layer of atoms that border its region. This is due to the specific neighborhood structure that consists of 4 neighbors which we have assumed in our Ising model (see Section 2). If the geometry were different (e.g., more dimensions, leading to more neighbors; or deeper penetration of influence) then we might need to check more atoms.

After exchanging information about their produced histories in step (c), the PEs detect inconsistencies. Any boundary flip generated at the initial iteration causes inconsistencies in neighbors, because of the canonical assumption that there were no flips in boundaries. The communication in step (c) leads the PEs to have new assumptions about the histories of their neighbors, in place of the canonical assumption. Subsequent iterations, first step. After the PEs synchronize in step (d), they execute step (a) again. The first important distinction of this parallel procedure from its sequential prototype is that the feeding sequence $\left(U_{1}, V_{1}\right),\left(U_{2}, V_{2}\right), \ldots$ of random numbers at the subsequent iterations has to be the same as at the first iteration. No new random sample $U_{i}$ or $V_{i}$ in place of the previously generated one should be produced. This is in keeping with the notion given at the end of Section 4 that these numbers are viewed as presimulated, and need to be used in order.

As in the first iteration, each old $U_{i}$ is employed to generate $\tau_{i}$ and each old $V_{i}$ is employed to select atom $v_{i}$ for the flip. Because of different boundary conditions, the resulting $\tau_{i}$ and selected $v_{i}$ will generally differ from those computed at the previous iteration.

Moreover, the second main distinction between parallel and serial algorithms is that computing $\tau_{i}$, given $\tau_{i-1}$ and given the system state at time $\tau_{i-1}$, is not as straightforward as simply applying (3.4). The complication arises because spins in the neighborhood may flip during time interval $\left(\tau_{i-1}, \tau_{i}\right)$, say at time $t_{b}$, which we call a break point time, $\tau_{i-1}<$ $t_{b}<\tau_{i}$

The class membership of some atoms hosted by the PE may change at time $t_{b}$ as a result. This in turn may change some $N_{i}$ and hence may change the $\Lambda$ computed by (3.3) and used in (3.4). The presence of a break point $t_{b}$ inside interval $\left(\tau_{i-1}, \tau_{i}\right)$, and the change of $\Lambda$ at $t_{b}$, makes formula (3.4) unusable as it is.

A more general method adjusts $\tau_{i}$ so as to satisfy the following equation

$$
\int_{\tau_{i-1}}^{\tau_{i}} \Lambda(t) d t=-\log U_{i} .
$$

Solving (5.2) for $\tau_{i}$ is easily accomplished since $\Lambda(t)$ changes in time in a piecewise constant fashion. One way of solving (5.2) is, with self-evident notation, to order the break points from $\tau_{b, 0}=\tau_{i-1}$ to the maximum $\tau_{b, m}=t_{c}+T_{\max }$, and to compute for $1 \leq i \leq m$ the sums

$$
\begin{aligned}
b_{k} & =\sum_{j=1}^{k}\left(\tau_{b, j}-\tau_{b, j-1}\right) \\
& =\int_{\tau_{i-1}}^{\tau_{b, k}} \Lambda(t) d t
\end{aligned}
$$


If $-\log U_{i}>b_{m}$ then $\tau_{i}>t_{c}+T_{\max }$, and we are done. If $-\log U_{i} \leq b_{m}$, then since the $b_{k}$ are monotone increasing, we easily find $k$ such that

$$
b_{k-1}<-\log U_{i} \leq b_{k} .
$$

Given $k$, we have (analogously to equation (3.4))

$$
\tau_{i}=\tau_{b, k}+\frac{-\log U_{i}-b_{k-1}}{\Gamma\left(\tau_{b, k-1}\right)} .
$$

Another, more efficient, way to solve (5.2) for $\tau_{i}$, is to generate the $b_{k}$ one at a time until equation (5.5) is satisfied.

Another distinction from sequential simulation is a possible need for additional random numbers. The need may arise as a result of change of spin flip times $\tau_{i}$ at iterations. A subsequent iteration may fit more spin flips, or fewer spin flips, within the time interval $\left(t_{c}, t_{c}+T_{\max }\right)$. Recall that a presimulated list will have the random numbers drawn in order, and so additional random numbers must be drawn after the original ones are used. In particular, the random number $U_{m+1}$ that caused $\tau_{m+1}$ to exceed $T_{\max }$ would be the first one used if more random numbers are needed. If fewer flips are needed, we save unused random numbers for possible use in future iterations or subsequent time steps.

Iterations of the synchronous relaxation steps a, b, c, and d continue until all PEs realize that the assumptions they made at the beginning of an iteration agree with the information they receive at the iteration's end; that is, the times $\tau_{i}$ and flipped atoms $v_{i}$ are the same as in the previous-iteration assumption and this hold for all $\tau_{i}<T_{\max }$. This signals that it is time to advance the committed time to $t_{c}=T_{\max }$ for a new time step, and to continue with a new round of iterations if necessary.

Subsequent steps. The only difference of this situation from those already described is in defining initial conditions. Whereas $\tau_{0}=0$ at the first time advancement step and the initial system state is that at time 0 , for the following steps, the initial state of the subsystem hosted by a PE is the state resulting from the last simulated event at the previous time advancement step.

Let $m$ be the index of the last spin flip simulated in the previous step. Using the notation above, the last event if any of the previous step was $\left(\tau_{m}, v_{m}\right)$. If, by chance, there were no events until now, then $m=0$, $\tau_{m}=0$, and $v_{m}$ is undefined. Redefining notations for the current time step, the old $\tau_{m}$ becomes the $\tau_{0}$ for this time step. This $\tau_{0}$ is used in this time step in the same way as the $\tau_{0}$ of the first time step was used. In particular, the $\tau_{1}$ can be found using either formula (3.4) or by solving equation (5.2) with respect to $\tau_{i}$, for $i=1$.

What we called $U_{i}$ and $V_{i}$ at the previous time step we now rename to $U_{i-m}$ and $V_{i-m}$; so $U_{m+1}$ becomes $U_{1}$, etc. Again, from viewing random numbers as coming from a presimulated list, any remaining random samples $V_{m+1}, U_{m+2}, V_{m+2}, \ldots$, must be reused before additional random sampling is done. They are to be renamed in the new time step as $V_{1}, U_{2}, V_{2}, \ldots$. respectively.

An alternate approach to subsequent steps. For Ising simulations, properties of Poisson random variables enable us to wipe clean the slate, empty event lists of all PEs, and start afresh at the time $t_{c}+T_{\max }$. That is, we need not keep any knowledge of tentative events generated at previous steps. New random numbers may be generated at this time, taken later from a presimulated list than any up until now. This procedure may not be desirable; we simply wanted to point out that for Ising simulations it leads to unbiased simulations.

\section{Efficiency}

One of the nicest attributes of our algorithm is that it is provably efficient. In this section we outline the assumptions that go into the proof, and give a statement of the result. The detailed proofs can be found in the forthcoming paper [13].

The efficiency result is asymptotic as $N$, the number of PEs, becomes large. Our method is to bound the probabilistic distribution of the number of iterations needed to simulate one step of the algorithm. In addition, we bound the distribution of the amount of work needed to simulate each iteration. We also bound from below the average number of events simulated in each step. These bounds require a particular choice of $T_{\max }$ as a function of $N$, and also require some regularity of the model being simulated as $N$ increases. They also require that the load of simulated events remains balanced among the PEs as $N$ grows.

Specifically, suppose that the temperature is bounded away from zero during the entire time interval being simulated, and that $\lambda$ is bounded and is bounded away from zero, so that the ratio between the largest and smallest Poisson rates in the system 
remains bounded. The blocks of PEs may change size as $N$ increases; we suppose that the ratio between the number of atoms hosted by the different PEs remains bounded, that the ratio of the number of boundary atoms between any two PEs also remains bounded, and that the number of neighbors of any PE remains bounded, all independent of $N$. We also suppose that the number of boundary atoms per $\mathrm{PE}$ grows no faster than $\log N$. We choose $T_{\max }$ so that for each $\mathrm{PE}$, the number of boundary atoms times $T_{\max }$ is within a constant factor of $\log N$. Clearly, this is possible because of the preceding assumptions. This choice ensures that the maximum and minimum expected number of events occurring among the boundary atoms of any PE during a time interval of length $T_{\max }$ is of order $\log N$.

The preceding assumptions relate to the system being simulated. Our efficiency result also requires some assumptions about the machine doing the simulation. We assume that synchronization can be done in no more than order $\log N$ time. We assume that communicating $x$ events from one PE to a neighboring PE takes no more than a constant times $x+\log N$, and that this can be done in parallel, so that if $x$ is the maximum number of events to be communicated from any $\mathrm{PE}$ to its neighbor, then all communication can be done in no more than a constant times $x+\log N$ time. We suppose that the speed of each $\mathrm{PE}$ is within a constant factor of the speed of any other PE; speed is the number of operations per unit of time. We suppose that the unit of time is chosen so that the speeds of the PEs is constant as $N$ increases.

Now for a bit of notation. For any iteration, we let $f$ represent the maximum total number of events that occur at the boundary atoms of a PE. That is, $f$ is the maximum number of events that need to be communicated at each iteration. We let $g$ denote the number of iterations to complete one step. Our assumptions show that the amount of time required to complete a simulation cycle is bounded above by a constant times $f g$. Note that $f, g$, and hence $f g$ are random variables. We let $K$ denote the maximum size of any set consisting of a PE, its neighbors, and their neighbors. $K$ is bounded by the assumption that the set of neighbors is bounded in size.

We write $x \leq_{s} y$ for real-valued random variables $x$ and $y$ if

$$
\mathbb{P}(x>z) \leq \mathbb{P}(y>z)
$$

holds for any real number $z$.

Under the assumptions delineated in the preceding section, we derive the following results.

Theorem 1 If the average number of events in a PE during an iteration is $\log N$, then for each constant $C>1$

$$
f \leq_{s} C e \log N+Y,
$$

where $Y$ is a geometrically distributed random variable with parameter $1 / C$.

In particular, this theorem shows that $E(f) \leq$ $C e \log N+1 /(C-1)$, and $\sqrt{\operatorname{Var}(f)} \leq C e \log N+$ $2 /(C-1)^{2}$.

We also find the following.

Theorem 2 If the average number of events in a PE during an iteration is $\log N$, then for each constant $C>1$ we find

$$
g \leq_{s} C e K \log N+X
$$

where $X$ is a geometrically distributed random variable with parameter $1 / C$.

In particular, this theorem shows that $E(g) \leq$ $C e K \log N+1 /(C-1)$ and $\sqrt{\operatorname{Var}(g)} \leq C e K \log N+$ $2 /(C-1)^{2}$.

Combining these two theorems, we can bound the average time for a complete simulation cycle of length $T_{\max }$. We may find a constant $c$ such that, for large enough $N$, by Schwarz's inequality,

$$
E(f g) \leq \sqrt{E\left(f^{2}\right) E\left(g^{2}\right)} \leq(c \log N)^{2} .
$$

Since our assumptions show that the amount of time to complete a simulation cycle is no more than proportional to $f g$, equation (6.4) gives our bound on the mean real time required to simulate time $T_{\max }=$ $\log N$. Furthermore our assumptions show that in time $T_{\max }=\log N$ we expect to have to within a constant factor $N T_{\max }\left|G_{i}\right| \lambda p_{i}$ events simulated. Therefore, the number of events simulated per unit time is, to within a factor $c$,

$$
\frac{\# \text { events }}{\text { unit time }}=c \frac{N \log N}{(\log N)^{2}}=c \frac{N}{\log N},
$$

which is the efficiency estimate we promised.

We summarize our efficiency result as a theorem.

Theorem 3 Under the assumptions, the efficiency of the simulation is at least of order $1 / \log N$ as $N \rightarrow$ $\infty$. Specifically, let $\tau(N, t)$ be the amount of real time it takes to simulate an interval of time $(0, t)$ with $N$ processors. Then there is a constant $B$ such that

$$
\frac{\tau(N, t)}{(t+1) \log N} \leq B
$$


We note that it can be shown that our scaling is optimal, in the sense that by taking a function $T_{\max }=r(N)$ in place of $\log N$, where either $r(N) / \log N \rightarrow \infty$ or $r(N) / \log N \rightarrow 0$ as $N \rightarrow \infty$, gives strictly worse efficiency. The details of all these results are in the full paper [13].

\section{References}

[1] S. Borst, S. Grandhi, Kahn, K. Kumaran, B.D. Lubachevsky, D. Sand Wireless Simulation and Self-Organizing Spectrum Management. Bell Labs Tech. J., V.2, No.3, 1997, pp.81-98.

http://www.lucent.com/minds/techjournal /summer_97/pdf/paper06.pdf

[2] A.B. Bortz, M.H. Kalos, J.L. Lebowitz, "A New Algorithm for Monte Carlo Simulation of Ising Spin Systems," J. Comput. Phys. 17, No. 1, pp. 10-18, 1975.

[3] K.M Chandy and R. Sherman, "Space-Time and Simulation," Proc. SCS Multiconference on Distributed Simulation, March 1989, Tampa, Florida, (SCS Simulation Series, v.21, No.2), pp.53-57.

[4] S. Eick, A.G. Greenberg, B.D. Lubachevsky, and A. Weiss, "Synchronous Relaxation for Parallel Simulation with Applications to Circuit Switched Networks," ACM Trans. On Modeling And Computer Simulation 3, No. 4, pp. 287-314, 1993.

[5] R.J. Glauber, "Time-Dependent Statistics of the Ising Model," J. Math. Physics 4, 2, pp. 294-307, 1963.

[6] P. Heidelberger and D.M. Nicol, "Conservative Parallel Simulation of Continuous Time Markov Chains Using Uniformization," IEEE Trans. Parallel Distr. Syst., 4, No. 8 (1995), pp. 906-921.

[7] F. Ising, "Beitag zur theorie des ferromagnetismus," Z. Physik, 31, pp. 253-258, 1925.

[8] D. Jefferson, "Virtual Time," ACM TOPLAS, 7 , No. 3, 1985, pp.404-425.

[9] G. Korniss, G. Brown, M.A. Novotny, and P.A.Rikvold, Hard simulation problems in the modeling of magnetic materials: Parallelization and Langevin micromagnetics," in Computer Simulation Studies in Condensed Matter Physics XI, D..P. Landau and H.-B. Schuttler eds. (Springer-Verlag, New York, 1998)

http://xxx.lanl.gov/abs/cond-mat/9803118

[10] P.B. Linhart, B.D. Lubachevsky, R. Radner, and M.J. Meurer, "Friends and Family' and Related Pricing Strategies," Proc. 2nd Russian-Swedish Control Conference, Aug. 1995, St.Petersburg State Technical University, Russia, p. 192-196.

[11] B.D. Lubachevsky, "Efficient Parallel Simulations of Asynchronous Cellular Arrays," Complex Systems 1 (1987), pp. 1099-1123.

[12] B.D. Lubachevsky, "Efficient Parallel Simulations of Dynamic Ising Spin Systems," J. Computational Phys. 75, No. 1, pp. 103-122, 1988.

[13] B. Lubachevsky and A. Weiss, "Efficiency study of a new parallel Ising spin simulation algorithm," In preparation.

[14] N.C. Metropolis, A.W. Rosenbluth, M.N. Rosenbluth, A.H. Teller, and E. Teller, "Equation of State Calculations by Fast Computing Machines," J. Chemical Physics 21, No. 6, 1953.

[15] D.M. Nicol and P. Heidelberger, "Optimistic Parallel Simulation of Continuous Time Markov Chains Using Uniformization," Journ. Parallel and Distr. Comp., 18, no4, pp.395-410, 1993.

[16] P. Nielaba, V. Privman, and J.-S. Wang, "Irreversible Multilayer Adsorption," in Computer Simulation Studies in Condensed-Matter Physics V- (D.P. Landau, K..K Mon, and HB. Schüttler, Eds.), Proceedings in Physics, Vol 76 (Springer-Verlag, Berlin, 1993), p.143. 